\title{
Factores socioeconómicos y engagement académico en estudiantes universitarios en contextos de pandemia por SARS-CoV-2
}

Socioeconomic factors and academic engagement in university students in contexts of the SARS-CoV-2 pandemic

Fatores socioeconômicos e engajamento acadêmico em estudantes universitários em contextos da pandemia SARS-CoV-2

Vidnay Noel Valero Ancco

vvalero@unap.edu.pe https://orcid.org/0000-0002-7980-0339

Universidad Nacional del Altiplano, Perú

Miryam Pari Orihuela mpari@unap.edu.pe https://orcid.org/0000-0002-8287-9498 Universidad Nacional del Altiplano, Perú
Katty Maribel Calderon Quino

kattycalderon@unap.edu.pe https://orcid.org/0000-0002-1356-3182

Universidad Nacional del Altiplano, Perú

Uriel Arpasi Mamani

urielarpasi@gmail.com

https://orcid.org/0000-0003-3986-3279

I.E.S. Mariano Melgar Ccallata El Collao Ilave, Perú

Artículo recibido en junio 2021, revisado en julio 2021, arbitrado en agosto 2021 y publicado en septiembre 2021

\section{RESUMEN}

La presente investigación se guio por el objetivo de establecer si los factores socioeconómicos como el acceso a la tecnología, servicio de internet y la condición laboral se relacionan con el engagement académico (EA) en estudiantes universitarios en contextos de educación remota. El trabajo se enmarca en el tipo no experimental con diseño correlacional transversal y explicativo. La muestra fue conformada por 253 estudiantes de la Facultad de Ciencias de la Educación, específicamente 222 damas y 31 varones, seleccionados por un criterio no probabilístico. Para recoger la información se empleó la técnica de la encuesta a través del cuestionario sociodemográfico y el cuestionario de Utrecht Work Engagement Scale (UWES-17) administrado vía Google Forms. Los resultados encontrados indican que no existe relación entre el acceso a la tecnología, servicio de internet y la condición laboral con el EA en universitarios en contextos de educación remota.

Palabras clave: Engagement académico; Educación superior; Estudiantes que trabajan; Factores socio-económicos; Satisfacción en los estudios

\section{ABSTRACT}

This research was guided by the objective of establishing whether socio-economic factors such as access to technology, internet service and employment status are related to academic engagement (EA) in university students in remote education contexts. The work is framed in the non-experimental type with cross-sectional and explanatory correlational design. The sample was made up of 253 students from the Faculty of Education Sciences, specifically 222 ladies and 31 men, selected by a non-probabilistic criterion. To collect the information, the survey technique was used through the sociodemographic questionnaire and the Utrecht Work Engagement Scale questionnaire (UWES-17) administered via Google Forms. The results found indicate that there is no relationship between access to technology, internet service and the labor condition with the EA in university students in remote education contexts.

Key words: Academic engagement; Higher education; Working students; Socio-economic factors; Satisfaction in studies

\section{RESUMO}

Esta pesquisa foi orientada pelo objetivo de estabelecer se o acesso à tecnologia e serviços de internet; e a condição de trabalho estão relacionadas ao engajamento acadêmico (EA) em estudantes universitários em contextos de educação remota. O trabalho é enquadrado no tipo não experimental com desenho correlacional transversal e explicativo. $\mathrm{O}$ trabalho é enquadrado no tipo não experimental com desenho correlacional transversal e explicativo. A amostra foi composta por 253 alunos da Faculdade de Ciências da Educação, especificamente 222 mulheres e 31 homens, selecionados por critério não probabilístico. Para coletar as informações, a técnica de pesquisa foi utilizada por meio do questionário sociodemográfico e do questionário Utrecht Work Engagement Scale (UWES-17) administrado via Formulários do Google. Os resultados encontrados indicam que não há relação entre o acesso à tecnologia, $\mathrm{o}$ serviço de internet e a condição de trabalho com a EA em estudantes universitários em contextos de educação remota.

Palavras-chave: Envolvimento acadêmico: Ensino superior; Estudantes que trabalham; Fatores socioeconômicos; Satisfação nos estudos 


\section{INTRODUCCIÓN}

El sistema universitario en el último quinquenio ha sufrido modificaciones, desde la implementación de una nueva ley universitaria, que tiene mayores exigencias en cuanto a calidad educativa $y$ competitividad (Ruiz y Manrique, 2016), así mismo la instauración de la Superintendencia Nacional de Educación Superior Universitaria (SUNEDU) encargada de la administración del licenciamiento de las universidades del país (Zegarra, 2019), por otro lado está el Sistema Nacional de Evaluación, Acreditación y Certificación (SINEACE) cuyo objetivo es garantizar que las universidades ofrezcan un servicio de calidad. Si bien es cierto a través de todos estos sistemas se busca mejorar las funciones de las universidades como la formación integral, investigación (Valero, 2021b), proyección social entre otros (Martí et al., 2018), poniendo énfasis en procesos de autoevaluación, mejora continua (SINEACE, 2017), infraestructura, recursos económicos, entre otros.

Uno de los principios que manifiesta la Ley Universitaria es el interés superior del estudiante (Ley Universitaria - Ley 30220, 2014), muchas de las actividades que emprende la Universidad y los Programas Académicos que la componen se han focalizado en este principio procurando satisfacer las necesidades educativas de los estudiantes en las aulas universitarias (Ruiz y Manrique, 2016); sin embargo desde la llegada de la pandemia por SARS-CoV-2 ha originado un retraso en el avance que se venía realizando, provocando cambios abruptos en las formas de enseñanza en la universidad (Guarnizo, 2021). Sobre todo, porque los estudiantes ya no tienen acceso a los recursos tecnológicos que se encuentran en las universidades, como son los equipos de cómputo, equipos de laboratorio, bibliotecas y servicio de internet gratuito, que permitía desarrollar con normalidad sus competencias académicas, estando muchos lejos de estos servicios se han visto perjudicados, incluso han abandonado los estudios por la precariedad en la que se encuentran (Palacio et al., 2020; Pequeño et al., 2020).

De lo descrito se asume que varios estudiantes se han visto forzados a abandonar los estudios, otros a compartir sus actividades académicas con el trabajo o cuidado de sus familiares y también por encontrarse en zonas rurales donde no se cuenta con servicios como electricidad o internet (Pérez et al., 2020). Todos estos factores pueden convertirse en causas de disminución de la dedicación al estudio y compromiso académico de los estudiantes, lo que en la literatura científica se conoce como el engagement académico EA.

Actualmente las investigaciones de EA, se sustentan en la psicología positiva, que se aboca al estudio del buen funcionamiento y las fortalezas que posee y utiliza la persona para afrontar situaciones de estrés (Caballero, Hederich, et al., 2015). Dentro de ese marco conceptual se entiende el EA como el esfuerzo que hacen los estudiantes con el fin de obtener resultados académicos positivos (Cavazos y Encinas, 2016), también se le considera como las acciones que efectúan y en las que se involucran los estudiantes con el fin de alcanzar el éxito académico (Aspeé et al., 2018), así mismo se define como un estado psicológico positivo que asume un estudiante para afrontar los estudios (Caballero, Gonzales, et al., 2015) y también como los sentimientos, comportamientos y creencias en relación a su ambiente de estudio (Coelho y Dell'Aglio, 2018). Por otra parte, Tacca et al. (2021), manifiestan que el EA es un elemento esencial para promover aprendizajes, mejores rendimientos y sobre todo bienestar y disfrute de los alumnos en sus estudios. 
El EA se conforma por tres dimensiones: el vigor, la dedicación y la concentración (Arias et al., 2020; Daura et al., 2020; Pérez et al., 2021; Schaufeli y Bakker, 2004). El Vigor se define como los altos niveles de energía que pone el estudiante para la realización de sus tareas y actividades de estudio (Rigo et al., 2020), también se define como el deseo de esforzarse que tiene el estudiante (Gil y Cruz, 2018).

Por otro lado, se tiene la dimensión dedicación que se concibe como elevados niveles de entusiasmo, involucramiento y orgullo que asume el estudiante en el proceso de su formación y realización de tareas (Rodríguez et al., 2020), este aspecto puede verse afectado por características económicas. En ese sentido Garzon y Gil (2017) señalan que los estudiantes que combinan sus estudios con el trabajo tienen mayores inconvenientes para cumplir tareas académicas provocando la postergación de estas. Y finalmente la dimensión absorción que se conceptualiza como un estado positivo de concentración, en el que los estudiantes tienen un disfrute intrínseco en la ejecución de sus tareas, además de enfocarse plenamente en el desarrollo de estas con una armonía mental y corporal (Portalanza et al., 2017).

Diversas investigaciones han evidenciado que el EA, están relacionado con diversos factores socioemocionales, sociodemográficos, y factores académicos (García y Colás, 2020; Gómez et al., 2015; Maluenda et al., 2020; Martínez et al., 2019), dentro de ellos predomina, la edad, la condición laboral, la situación sentimental de los estudiantes, la condición académica de los estudiantes, ya sean regulares u observados (Valero, 2021a); estos factores en su mayoría fueron analizados en contextos normales de educación presencial. La relevancia del presente estudio recae en el contexto de pandemia y confinamiento que se ejecuta, siendo el objetivo principal identificar si los factores socioeconómicos como el acceso a la tecnología, servicio de internet y la condición laboral se relacionan con el EA en estudiantes universitarios en contextos de educación remota, producto de la pandemia por Sars-Cov-2.

\section{MÉTODO}

El estudio se ejecutó en la Universidad Nacional del Altiplano de Puno, ubicada en la ciudad lacustre de Puno, Perú a 3826 msnm. La investigación se elaboró durante el primer semestre del año académico 2021 en los meses de abril y mayo.

La investigación corresponde al tipo no experimental, con diseño correlacional además es de carácter transversal, descriptivo y explicativo. La población del estudio está conformada por 253 estudiantes de la Facultad de Ciencias de la Educación, específicamente 222 mujeres y 31 varones, comprendidos entre 18 a 30 años de edad, seleccionados por un criterio no probabilístico. Para recoger la información se empleó la técnica de la encuesta a través de un cuestionario electrónico de Google Forms, dividido en dos partes. La primera parte compuesta por ocho preguntas recoge las características sociodemográficas de los investigados. En la segunda parte se utilizó el cuestionario de Utrecht Work Engagement Scale (UWES-17) de (Schaufeli y Bakker, 2004) constituido por 17 preguntas y evalúa los tres aspectos que componen el EA, como son el vigor, la dedicación y la absorción a través de una escala de Likert.

El procesamiento de datos se realizó mediante la estadística descriptiva por medio de la 
presentación de tablas de frecuencia y también se utilizó la estadística inferencial aplicando la prueba no paramétrica de chi cuadrado, para evidenciar la relación o asociación de las variables de estudio.

\section{RESULTADOS Y DISCUSIÓN}

\section{Resultados del nivel de engagement en la dimensión vigor}

Respecto a la dimensión vigor del EA se obtuvo que 124 estudiantes que representan el $49 \%$ de la población se caracteriza por un nivel promedio; también se tiene 72 estudiantes que representan el $28.5 \%$ en un nivel muy bajo, también se encontró 47 estudiantes que representan el $18.6 \%$ con un nivel bajo y solamente un $4.2 \%$ acumulado en los niveles de alto y muy alto (Tabla 1 ).

Tabla 1. Resultados de la dimensión vigor del EA en estudiantes universitarios 2021.

\begin{tabular}{lccc}
\hline Escala & Frecuencia & Porcentaje & Porcentaje acumulado \\
\hline Muy bajo & 72 & 28.5 & 28.5 \\
\hline Bajo & 47 & 18.6 & 47.0 \\
Medio & 124 & 49.0 & 96.0 \\
Alto & 7 & 2.8 & 98.8 \\
Muy alto & 3 & 1.2 & 100.0 \\
\hline Total & $\mathbf{2 5 3}$ & $\mathbf{1 0 0 . 0}$ & \\
\hline
\end{tabular}

Estos resultados manifiestan que la mayor cantidad de estudiantes investigados se localizan entre el nivel promedio, bajo y muy bajo de vigor en relación al EA. Estos resultados coinciden con lo encontrado por (Nájar, 2017) en estudiantes de una universidad Privada donde el mayor porcentaje de la población también se encontró en un nivel promedio de EA; a diferencia de (Gonzales, 2017) quien en su estudio encontró mayormente estudiantes en los niveles promedio y alto. La dimensión vigor de EA refleja la voluntad de los estudiantes por alcanzar los objetivos académicos propuestos, la energía que ellos ponen en la realización de las actividades académicas; sin embargo, esta no se relaciona con los niveles de rendimiento académico, es decir poseer altos niveles de vigor no es garantía de que los estudiantes tengan buenos promedios en sus calificativos.

\section{Resultados del nivel de engagement en la dimensión dedicación}

En la dimensión dedicación del EA se encontró 115 estudiantes que equivalen el $45.5 \%$ de la población se caracteriza por poseer un nivel medio de dedicación, también se tiene 50 estudiantes que representan el $19.8 \%$ de la población se caracteriza por un nivel muy alto de dedicación, por otro lado, tiene 36 estudiantes que representan el 14.2\% de la población que se caracteriza por poseer un nivel alto. En los niveles bajo y muy bajo se tiene un acumulado de $20.6 \%$ (Tabla 2 ). 
Tabla 2. Resultados de la dimensión dedicación del EA en estudiantes universitarios 2021.

\begin{tabular}{lccc}
\hline Escala & Frecuencia & Porcentaje & Porcentaje acumulado \\
\hline Muy bajo & 26 & 10.3 & 10.3 \\
\hline Bajo & 26 & 10.3 & 20.6 \\
Medio & 115 & 45.5 & 66.0 \\
Alto & 36 & 14.2 & 80.2 \\
Muy alto & 50 & 19.8 & 100.0 \\
\hline Total & $\mathbf{2 5 3}$ & $\mathbf{1 0 0 . 0}$ & \\
\hline
\end{tabular}

Estos resultados manifiestan que la mayoría de los estudiantes investigados se ubican entre el nivel promedio, alto y muy alto de dedicación en relación al EA. Estos resultados también coinciden con lo reportado por (Nájar, 2017) quien encontró niveles altos y muy altos de dedicación en estudiantes de una universidad privada, mientras que (Gonzales, 2017) encontró mayormente estudiantes con un nivel medio de dedicación de EA.

\section{Resultados del nivel de engagement en la dimensión absorción}

En relación a la dimensión absorción del EA se encontró 119 estudiantes universitarios que representan el $47 \%$ de la población se ubican en un nivel medio, también se tiene 57 universitarios que representan el $22.5 \%$ con un nivel bajo y 22 estudiantes que representan un $8.7 \%$ con un nivel muy bajo. En el nivel alto se encontró 39 estudiantes y en el nivel muy alto 16 estudiantes haciendo un porcentaje acumulado de $21.7 \%$ (Tabla 3 ).

Tabla 3. Resultados de la dimensión absorción del EA en estudiantes universitarios 2021.

\begin{tabular}{lccc}
\hline Escala & Frecuencia & Porcentaje & Porcentaje acumulado \\
\hline Muy bajo & 22 & 8.7 & 8.7 \\
\hline Bajo & 57 & 22.5 & 31.2 \\
Medio & 119 & 47.0 & 78.3 \\
Alto & 39 & 15.4 & 93.7 \\
Muy alto & 16 & 6.3 & 100.0 \\
\hline Total & $\mathbf{2 5 3}$ & $\mathbf{1 0 0 . 0}$ & \\
\hline
\end{tabular}

Estos resultados manifiestan que la mayoría de la población investigada se encuentra entre el nivel promedio, alto y bajo de absorción en relación al EA. Coincidentemente (Gonzales, 2017; Nájar, 2017) encontraron niveles medios y altos de absorción en estudiantes universitarios.

\section{Resultados del nivel de engagement en estudiantes universitarios}

Respecto a los resultados del nivel de EA en los estudiantes universitarios se encontró que 132 estudiantes que representan el $52.2 \%$ de la población se caracterizan por un nivel medio de EA, 
por otro lado, se tiene 49 estudiantes universitarios que representan el $19.4 \%$ del total con un nivel de EA bajo y un $12.6 \%$ con un nivel muy bajo. En el nivel alto de EA se tiene 25 estudiantes y en el nivel muy alto 15 estudiantes haciendo un porcentaje acumulado de $15.8 \%$ del total de la población (Tabla $4)$.

Tabla 4. Resultados del nivel de engagement académico en estudiantes universitarios 2021.

\begin{tabular}{lccc}
\hline Escala & Frecuencia & Porcentaje & Porcentaje acumulado \\
\hline Muy bajo & 32 & 12.6 & 12.6 \\
\hline Bajo & 49 & 19.4 & 32.0 \\
Medio & 132 & 52.2 & 84.2 \\
Alto & 25 & 9.9 & 94.1 \\
Muy alto & 15 & 5.9 & 100.0 \\
\hline Total & $\mathbf{2 5 3}$ & $\mathbf{1 0 0 . 0}$ & \\
\hline
\end{tabular}

De estos resultados se asume que la mayoría de la población está en un nivel promedio de EA con proyecciones a los niveles bajo y muy bajo. Esto coincide con lo expresado por (Davey, 2016; Nájar, 2017) quiénes encontraron que la mayoría de estudiantes se encuentra en un nivel promedio con una proyección hacia el nivel alto. A diferencia de (Belito, 2020), quien en su investigación encontró predominancia del nivel bajo de EA y explica que las variables que inciden mayormente en estos resultados son la procastinación y el estrés académico de los estudiantes. Por otro lado Gonzales (2017) hace referencia a la relación que tiene el EA con la inteligencia emocional (IE) y el desempeño académico (DA), encontrando relación entre estas variables y explica que el EA y la (IE) son factores que influyen notablemente en el (DA) de los estudiantes universitarios.

Por otra parte (Davey, 2016), indica que el EA se relaciona con la variable felicidad, encontrando que la satisfacción con la vida y la realización personal se relacionan estrechamente con las dimensiones vigor y dedicación del EA. Así mismo el EA tiene efectos en la autoeficacia académica de los estudiantes (Carbajal, 2020). Todas estas investigaciones revelan que el proceso de formación de los estudiantes, no solo se deben centrarse en aspectos académicos, sino que se debe tener en cuenta el cómo se sienten los estudiantes y otros factores que se relacionan estrechamente con el EA, como es la procrastinación, el estrés, la felicidad que se traducirán en los resultados del (DA) de los estudiantes. Por consiguiente, es un error querer mejorar los niveles de (DA) de los estudiantes, sin mirar los factores que influyen en él.

\section{Resultados de la relación de la condición laboral con el engagement académico}

En cuanto a la relación de las variables condición laboral con el nivel de EA, dentro de los parámetros más resaltantes, se encontró 132 estudiantes con un nivel promedio de EA, de los cuales 81 estudiantes estudian y trabajan; y 51 estudiantes solo estudian. También se tiene 49 estudiantes en un nivel bajo EA de los cuales 30 estudian y trabajan; y 9 solo estudian. En el otro extremo se tiene a 15 estudiantes con un nivel muy alto de EA de los cuales 10 estudian y trabajan y 5 solo estudian. Estos datos avizoran la no existencia de relación entre las variables (Tabla 5). 
Tabla 5. Resultados de la contingencia de la condición laboral con el nivel de engagement académico en estudiantes universitarios 2021.

\begin{tabular}{lccc}
\hline \multirow{2}{*}{ Nivel de engagement } & \multicolumn{2}{c}{ Condición laboral } & Total \\
\hline Muy bajo & Estudia y trabaja & Solo estudia & 32 \\
Bajo & 23 & 9 & 49 \\
Promedio & 30 & 19 & 132 \\
Alto & 81 & 51 & 25 \\
Muy alto & 12 & 13 & 15 \\
Total & 10 & 5 & $\mathbf{2 5 3}$ \\
\hline
\end{tabular}

Aplicando la prueba estadística chi cuadrado para medir la asociación y dependencia de las variables, se obtuvo un valor de 3.554 con un valor de la significante de 0.470 que es mayor al valor propuesto $(0.470>0.05)$, por lo tanto, se asume la hipótesis estadística nula concluyendo que no existe asociación ni dependencia entre la condición laboral y el EA en estudiantes universitarios (Tabla $6)$.

Tabla 6. Resultados de la prueba estadística de relación de la condición laboral con el nivel de engagement académico en estudiantes universitarios 2021.

\begin{tabular}{lccc}
\hline \multicolumn{1}{c}{ Prueba } & Valor & gl & Sig. asintótica (2 caras) \\
\hline Chi-cuadrado de Pearson & $3.554 \mathrm{a}$ & 4 & .470 \\
Casos válidos & 253 & & \\
\hline
\end{tabular}

a. 0 casillas $(0,0 \%)$ han esperado un recuento menor que 5 . El recuento mínimo esperado es 5,75.

Resultados de la relación del acceso a tecnología e internet con el engagement académico

En cuanto a la relación de las variables acceso a la tecnología e internet con el nivel de EA se encontró que 57 estudiantes manifestaron tener acceso limitado a equipos tecnológicos y servicio de internet, pero tienen un nivel promedio de EA. Así mismo existen 52 estudiantes que manifestaron tener un acceso deficiente a tecnología e internet, pero se caracterizan por un nivel promedio de EA. También se encontró 23 estudiantes que tienen un acceso optimo a equipos tecnológicos e internet y su nivel de EA también es medio (Tabla 7). 
Tabla 7. Resultados de la contingencia del acceso a tecnología e internet con el nivel de engagement académico en estudiantes universitarios 2021.

\begin{tabular}{lcccc}
\hline \multirow{2}{*}{ Nivel de engagement } & \multicolumn{3}{c}{ Acceso a tecnología e internet } & \multirow{2}{*}{ Total } \\
\hline Muy bajo & Acceso limitado & Acceso deficiente & Acceso suficiente & \\
Bajo & 12 & 13 & 7 & 32 \\
Promedio & 21 & 19 & 9 & 49 \\
Alto & 57 & 52 & 23 & 132 \\
Muy alto & 13 & 10 & 2 & 25 \\
Total & 5 & 8 & $\mathbf{4 3}$ & 15 \\
\hline
\end{tabular}

Aplicando la prueba estadística chi cuadrado para medir la asociación y dependencia de las variables, se obtuvo un valor de 3.539 con un valor de la significante de 0.896 que es mayor al valor propuesto $(0.896>0.05)$; por lo tanto, se asume la hipótesis estadística nula, concluyendo que no existe asociación ni dependencia entre el acceso a tecnología e internet con el EA en estudiantes universitarios (Tabla 8).

Tabla 8. Resultados de la prueba estadística de relación entre el acceso a tecnología e internet con el nivel de engagement académico en estudiantes universitarios 2021.

\begin{tabular}{lccc}
\hline \multicolumn{1}{c}{ Prueba } & Valor & gl & Sig. asintótica (2 caras) \\
\hline Chi-cuadrado de Pearson & $3.539 \mathrm{a}$ & 8 & .896 \\
Casos válidos & 253 & & \\
\hline
\end{tabular}

a. 2 casillas $(13,3 \%)$ han esperado un recuento menor que 5 . El recuento mínimo esperado es 2,55.

Respecto a la asociación que se hace entre la situación laboral de los estudiantes con el EA, se obtiene que no existe asociación ni dependencia entre las variables esto es corroborado por (Caballero, et al., 2015), quien afirma que estudiantes que pagan sus estudios tuvieron mejores resultados académicos y esto se relaciona positivamente con el EA, por lo tanto no se puede afirmar que todos los estudiantes que trabajan y estudian a la vez tengan bajos niveles de EA, ya que la condición de autosostenerse puede provocar niveles de estrés y agotamiento en los estudiantes, pero no es un factor que determine la dedicación, vigor y absorción en cuanto a sus estudios, es más un gran porcentaje de universitarios que estudian y trabajan resultaron poseer altos niveles de EA, sobre todo en la dimensión de vigor y dedicación, ya que tienen claro el objetivo a cumplir.

Así mismo se entiende que el EA está estrechamente relacionado con el interés y la perseverancia que tienen los estudiantes por cumplir las metas establecidas a pesar de las dificultades que sopesan (Daura et al., 2020), esto se manifiesta en la circunstancia actual, en la que muchos estudiantes al transitar de una educación virtual a una educción remota, se vieron afectados por la carencia de 
equipamiento tecnológico en sus hogares y acceso continuo a los servicios de internet, lo cual no causó la existencia generalizada de niveles bajos de EA, si bien es cierto existe grupos poblacionales que atribuirían a este factor como causa de deserción y abandono escolar, también hay grupos en los que a pesar de las dificultades mantienen niveles altos de EA, esto corrobora la hipótesis de que no se relaciona las condiciones de acceso a tecnología e internet con la energía, fuerza dedicación y empeño que ponen los estudiantes en sus estudios universitarios (Caballero, 2006; Y. Gonzales et al., 2019).

\section{CONCLUSIONES}

El nivel de EA de los estudiantes universitarios en tiempos de pandemia por Sars-Cov-2 se encuentra en un nivel medio, en cuanto a la dimensión vigor la mayor cantidad de estudiantes universitarios se caracteriza por poseer un nivel medio con tendencia al nivel bajo, en relación a la dimensión dedicación, la gran mayoría se ubicaron en un nivel medio con tendencia a los niveles alto y muy alto y en la dimensión absorción se encontró estudiantes en un nivel medio. La existencia de un nivel medio en las dimensiones de vigor y absorción, se explica teóricamente por las situaciones de estrés que vienen atravesando producto de la pandemia, el aislamiento social, la transición de una educación presencial a una remota, la presencia de familiares afectados por el virus Sars-Cov-2, inclusive la condición de trabajar y estudiar ha causado agotamiento y cansancio para el cumplimiento de sus actividades académicas en los universitarios.

A diferencia de la dimensión dedicación, donde se obtuvo niveles medios con tendencia a niveles altos incluso muy altos, reflejan el entusiasmo por el estudio de los jóvenes y señoritas, el orgullo e involucramiento con sus actividades académicas que, a pesar de las circunstancias adversas, ellos mantienen. Esto se refleja en los resultados alcanzados, al no encontrar relación por ejemplo entre la situación laboral y el nivel de (EA) de los universitarios, es decir el hecho de trabajar y estudiar no es una condicionante para afirmar que el EA será bajo o contrariamente el hecho de solo estudiar no garantiza niveles altos de EA y por consiguiente desempeños académicos y calificativos altos. Los mismos resultados se obtuvieron al relacionar las variables acceso a tecnología e internet con el EA, encontrándose que no hay asociación ni dependencia de las variables, lo que explica que los estudiantes universitarios aun teniendo pésimas condiciones de acceso a tecnología y servicio de internet para sus clases virtuales, mantienen niveles promedios y altos en cuanto a la dedicación de sus estudios.

REFERENCIAS

Arias, D., Vera, M., Ramos, T., y Pérez, S. (2020). Engagement e Inteligencia emocional en estudiantes de una universidad privada en la ciudad de Arequipa. Propósitos y Representaciones, 8(1), e423. https://doi. org/10.20511/pyr2020.v8n1.423

Aspeé, J., González, J., y Cavieres, E. (2018). El Compromiso Estudiantil en Educación Superior como Agencia Compleja. Formación Universitaria, 11(4), 95-108. https://doi. org/10.4067/S0718-50062018000400095

Belito, F. (2020). Procrastinación y estrés en el engagement académico de los estudiantes de educación de la Universidad Federico Villareal, 2019 [Universidad Cesa Vallejo]. Recuperado de: https://bit.ly/3io33kB

Caballero, C. (2006). Burnout, engagement y rendimiento académico entre estudiantes universitarios que trabajan y aquellos que no trabajan. Revista Psicogente, 9(16), 11-27 Recuperado de: https://bit.ly/2ZPJQ57 
Caballero, C., Gonzales, O., y Palacio, J. (2015). Relación del burnout y el engagement con depresión, ansiedad y rendimiento académico en estudiantes universitarios. Salud Uninorte, 31(1), 59-69. Recuperado de: https://bit. ly/3utKsso

Caballero, C., Hederich, C., y Garcia, A. (2015). Relacion entre burnout y engagement académico con variables sociodemográficas y académicas. Psicología Desde El Caribe, 32(2), 254-267. Recuperado de: https://bit.ly/3Fi2SRP

Carbajal, C. (2020). Autoeficacia académica, compromiso y satisfacción con los estudios en estudiantes universitarios de Lima Metropolitana [Universidad San Martin de Porres]. Recuperado de: https://bit.ly/3iFBxiT

Cavazos, J., y Encinas, F. (2016). Influencia del engagement académico en la lealtad de estudiantes de posgrado: un abordaje a través de un modelo de ecuaciones estructurales. Estudios Gerenciales, 32(140), 228-238. https:// doi.org/10.1016/j.estger.2016.07.001

Coelho, C., y Dell'Aglio, D. (2018). Engajamento escolar: Efeito do suporte dos pais, professores e pares na adolescência. Psicologia Escolar e Educacional, 22(3), 621-629. https://doi. org/10.1590/2175-35392018038539

Daura, F., Barni, M., González, M., Assirio, J., y Lúquez, G. (2020). Evaluación del Compromiso académico y Grit. Fortalezas de carácter a desarrollar en estudiantes de postgrado. Revista Digital de Investigación En Docencia Universitaria, 14(1), e1172. https://doi. org/10.19083/ridu.2020.1172

Davey, K. (2016). Felicidad y engagement en estudiantes de educación superior que trabajan en Lima [Universidad Nacional Mayor de San Marcos]. Recuperado de: https://bit. ly/3AXAqC9

García, L., y Colás, P. (2020). Factores pedagógicos asociados con el compromiso de los universitarios con sus estudios. Formación Universitaria, 13(6), 181-190. https://doi. org/10.4067/S0718-50062020000600181
Garzon, A., y Gil, J. (2017). Gestión del tiempo y procrastinación en la educación superior. Universitas Psychologica, 16(3), 14. Recuperado de: https://bit.ly/2Y226r0

Gil, J., y Cruz, D. (2018). El burnout académico y el engagement en estudiantes de quinto año de Estomatología. Edumecentro, 10(4), 37-53. Recuperado de: https://bit.ly/3kT7CoC

Gómez, P., Pérez, C., Parra, P., Ortiz, L., Matus, O., McColl, P., Torres, G., y Meyer, A. (2015). Relación entre el bienestar y el rendimiento académico en alumnos de primer año de medicina. Revista Médica de Chile, 143(7), 930937. Recuperado de: https://bit.ly/3on0qDm

Gonzales, D. (2017). Engagement en el estudio, inteligencia emocional y rendimiento académico en estudiantes de la Carrera Profesional de Ingeniería Forestal de la UNSAAC, 2014 [Universidsad Nacional Mayor de San Marcos]. Recuperado de: https://bit.ly/3FgMh0P

Gonzales, Y., Da Cuña, I., Soto, M., y Alonso, A. (2019). Evolución del engagement académico en función de las variables sociodemográficas. Edunovatic: 3 rd Virtual International Conference On Education, Innovation an ICT, 659.

Guarnizo, A. (2021). Vicisitudes y retos pedagógicos en medio de la emergencia sanitaria. La formación médica en tiempos de COVID-19. Educación Médica, xxxx, 10-13. https://doi. org/10.1016/j.edumed.2021.01.008

Ley Universitaria - Ley 30220 (p. 35). (2014). Congreso de la República. Recuperado de: https://bit.ly/3D3iJBU

Maluenda, J., Flores, G., Varas, M., y Díaz, A. (2020). Comportamientos interpersonales del docente asociados al compromiso académico de estudiantes de primer año de Ingeniería. Revista de Estudios y Experiencias En Educación, 19(39), 145-161. https://doi.org/10.21703/ rexe.20201939maluenda8

Martí, J., Calderon, A., y Fernandez, A. (2018). La responsabilidad social universitaria en Iberoamérica: análisis de las legislaciones de 
Brasil, España y Perú. Revista Iberoamericana de Educación Superior, IX(24), 107-124. Recuperado de: https://bit.ly/3upzPqq

Martínez, I., Meneghel, I., y Peñalver, J. (2019). Does Gender Affect Coping Strategies Leading to WellBeing and Improved Academic Performance? Revista de Psicodidactica, 24(2), 111-119. https://doi.org/10.1016/j.psicod.2019.01.003

Nájar, M. (2017). Engagement Académico y Rendimiento Académico en Estudiantes de la Beca Vocación Maestro en una Universidad Privada. Arequipa, 2017 [Universidad Católica de Santa María]. Recuperado de: https://bit. ly/3onzLXg

Palacio, L., Vargas, J., y Monroy, S. (2020). Análisis bibliométrico de estudios sobre factores socioeconómicos en estudiantes universitarios. Educación y Educadores, 23(3), 355-375. https:// doi.org/10.5294/edu.2020.23.3.1

Pequeño, I., Gadea, S., Alborés, M., Chiavone, L., Fagúndez, C., Giménez, S., y Santa Cruz, A. B. (2020). Enseñanza y aprendizaje virtual en contexto de pandemia. Experiencias y vivencias de docentes y estudiantes de la Facultad de Psicología en el primer semestre del año 2020. InterCambios. Dilemas y Transiciones de La Educación Superior, 7(2), 150-170. https://doi. org/10.2916/inter.7.2.14

Pérez, M., Gasquez, J., Molero, M., Martos, A., Barragan, A., y Simon, M. (2021). Student burnout and engagement: Relationship with adolescent use of alcohol and attitudes towards authority. International Journal of Clinical and Health Psychology, 21(2), 100225. https://doi. org/10.1016/j.ijchp.2021.100225

Pérez, R., Martínez, M., y Mena, E. (2020). SarsCoV-2 en México y su efecto en los modelos educativos áulicos: una perspectiva crítica y reflexiva. RIDE Revista Iberoamericana Para $\mathrm{La}$ Investigación y El Desarrollo Educativo, 11(21). https://doi.org/10.23913/ride.v11i21.814

Portalanza, C., Grueso, M., y Duque, E. (2017). Propiedades de la Utrecht Work Engagement Scale (UWES-S 9): análisis exploratorio con estudiantes en Ecuador. Innovar, 27(64), 145-156. https://doi.org/10.15446/innovar. v27n64.62374

Rigo, R., Marques, J., y Corte, M. (2020). Engagement acadêmico no ensino superior: Premissa pedagogica para o desenvolvimento de competências transferíveis. Educação Em Revista, 36(e217239), 1-16. https://doi. org/10.1590/0102-4698217239

Rodríguez, S., Piñeiro, I., Regueiro, B., y Estévez, I. (2020). Intrinsic motivation and perceived utility as predictors of student homework engagement. Revista de Psicodidáctica, 25(2), 93-99. https:// doi.org/10.1016/j.psicoe.2019.11.001

Ruiz, C., y Manrique, J. (2016). Acreditación universitaria en las carreras de odontología en el Perú. 26(2), 85-91. Recuperado de: https://bit. ly/39Syhfe

Schaufeli, W., y Bakker, A. (2004). Bevlogenheid: een begrip gemeten 1. Gedrag \& Organisatie, 17(2), 89-112. Recuperado de: https://bit.ly/3m6lYl2

SINEACE. (2017). Modelo de acreditación para programas de estudio de educación superior universitaria. S. D. Técnicos (Ed.)

Tacca, D., Hernandez, L., Alva, M., y Romero, E. (2021). Propiedades psicométricas de una escala de compromiso académico en estudiantes universitarios peruanos. Rip, 2(23), 9-21. Recuperado de: https://bit.ly/3Am2oGu

Valero, V. (2021a). Actitud de los estudiantes universitarios ante las actividades extracurriculares. PURIQ, 3(1), 125-141. https://doi.org/10.37073/puriq.3.1.123

Valero, V. (2021b). La investigación formativa en la universidad. Revista Latinoamericana Ogmios, 1(1), 7-8. https://doi.org/10.53595/ rlo.2021.1.001

Zegarra, O. (2019). Modelo de licenciamiento de los programas de pregrado de Medicina en el Perú. 36(4), 301-308. Recuperado de: https://bit. ly/3ou $3 \mathrm{dKW}$ 